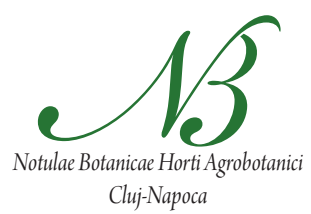

\title{
The Influence of Extraction Method on the Apparent Content of Bioactive Compounds in Romanian Allium spp. Leaves
}

\author{
Liliana GÎTIN, Rodica DINICĂ*, Raluca PARNAVEL \\ "Dunărea de Jos" University of Galati, 111 Domnească Street, 800201, Galați, Romania; rodinica@ugal.ro (*orresponding author)
}

\begin{abstract}
In order to characterize the composition of bioactive compounds in Allium spp., an important source of traditional foods, analyses of their physicochemical properties, total phenols, and flavonoid content were carried out using three of the most popular Romanian leaf varieties (Allium cepa var. 'Diamant', 'Rubiniu', and Allium ursinum L.). The highest levels of bioactive compounds were detected in the leaves of Allium ursinum L., $7.2730 \mathrm{mg}$ QE/ kg fresh plant and $27.8884 \mathrm{~g}$ GAE/100 g dry basis, respectively. This study suggests potential good uses of the fresh leaves of Romanian Allium spp. as condiments, ingredients or preservatives in the food industry.
\end{abstract}

Keywords: Allium cepa, Allium ursinum, flavonoids, total polyphenols

\section{Introduction}

Allium is the most representative genus of the Liliaceae family, which includes 700 species of widely distributed bulbous perennials and biennials (Najjaa et al., 2007; Tepe et al., 2005), and is an important economic genus due to the huge quantities of onions consumed. At present, $\mathrm{Al}$ lium spp. plants are considered the most important vegetables consumed fresh or in different cooked dishes in Europe, Asia, and America (Djurdjevic et al., 2004). In Romania, over the last year, significant changes took place in the vegetable market. In 2010, according to the Ministry of Agriculture, the production of dried onions was estimated at 362.3 thousand tones, and Romania was situated at third place according to statistics (FRD Center Market Report, 2011). Allium spp. plants are used as common foods, and for the treatment of many diseases (Lanzotti, 2006), due to their content of phytonutrients. In fact, the edible parts of Allium spp. plants are used for the treatment and prevention of a number of diseases: coronary heart disease (Gorinstein et al., 2007; Hiyasat et al., 2009; Siegel et al., 2004), cancer (Sengupta et al., 2004; Shukla and Kalra, 2007), obesity, diabetes, disturbances of the gastrointestinal tract, hyper-cholesterolemia, and inflammatory diseases (Hodge et al., 2002; Kalayarasan et al., 2009; Takahashi and Shibamoto, 2008).

Other researchers (Dini et al., 2008) have reported an increasing interest in Allium spp., both by the food industry and by scientists researching vegetables and plants, because of their strong antioxidant properties. These properties are due to many substances, including some vitamins, flavonoids, terpenoids, carotenoids, phytoestrogens, minerals, and volatiles compounds. Onion and wild garlic contain polyphenolic compounds. Flavonols and anthocy- anins are the main subclasses of flavonoids present in onions, the latter being found only in red onions (Rodrìgues et al., 2009, 2011). The actual number of polyphenols is estimated to be higher because they generally occur as glycosides, and the details of their sugar residues and binding forms show great variety (Miron et al., 2010); so it is important to determine the amounts of polyphenols in plants. Literature reports of polyphenols are difficult to compare due to the use of different extraction solvents and conditions (Santas et al., 2008). Furthermore, onions and the edible portions of wild garlic are important components of the Romanian people's diet, and are commonly consumed uncooked, so the loss of bioactive compounds is minimized, contributing to the health benefits of this food (Gennaro et al., 2002; Santas et al., 2008; Sellappan and Akoh, 2002).

The aim of this study consisted of identifying and characterizing bioactive compounds from Romanian Allium spp. leaves, as an important constituent of their traditional foods. For this purpose, three species of plants (white onion, red onion, and wild garlic leaves) were analyzed in order to assess their physicochemical characteristics, and total phenolic and flavonoid contents, as important polyphenolic compounds.

\section{Materials and methods}

\section{Plant samples}

The most popular varieties of Romanian fresh onion leaves (Allium cepa L. var. 'Diamant' (white onion), Allium cepa var. 'Rubiniu' (red onion) from the early hybrids group were kindly supplied by the Research and Development Society for Vegetables Buzău, Romania. Fresh $\mathrm{Al}$ lium ursinum $\mathrm{L}$. leaves were collected from the Bacau city 
94

forests in March 2011. All plant samples were free of external damage, hand selected, and chopped in the mortar for the following analyses.

\section{Chemicals and materials}

Gallic acid, quercetin, vanillin, and Folin-Ciocalteu's phenol reagent were obtained from Merck (Darmstadt, Germany). Petroleum ether, toluene, glacial acetic acid, and dichloromethane were purchased from Lach-Ner s.r.o (Brno, Czech Republic). Ethanol was purchased from Tunic Prod (Bucharest, Romania). Solutions of $7.5 \%$ sodium carbonate, $2.5 \% \mathrm{AlCl}_{3} \cdot \mathrm{H}_{2} \mathrm{O}$, and $10 \%$ sodium carbonate were freshly prepared. Ultrapure water (TKA Smart 2 Pure UV/UF system, Niederelbert, Germany) was used for the experiments.

Determinations of moisture, ash, oil, $\mathrm{pH}$ and titratable acidity

The recommended methods were adopted to determine the levels of moisture, ash and oil (AOAC, 2000). The moisture content was determined by drying around $5.0 \mathrm{~g}$ of the samples, at $105^{\circ} \mathrm{C}$, to a constant weight. Oil content was determined by the Soxhlet method (Extraction Unit 6, SER 148 model, VELP Scientifica, Italy). Crude fat was obtained by exhaustively extracting $10 \mathrm{~g}$ of each sample in a Soxhlet apparatus using petroleum ether (boiling point range $40-60^{\circ} \mathrm{C}$ ) as the solvent (Dini et al., 2008). The ash content was determined by incinerating 5 $\mathrm{g}$ of fresh leaves at $550-600^{\circ} \mathrm{C}$ until a homogeneous white ash without black points was obtained. The $\mathrm{pH}$ and titratable acidity were determined in accordance with RoldánMarín et al. (2009). After determination of $\mathrm{pH}$, the solution was titrated with $0.1 \mathrm{~N} \mathrm{NaOH}$ to $\mathrm{pH} 8.1$, and the results were expressed as percentage of citric acid [ $\mathrm{g}$ of citric acid/per $100 \mathrm{~g}$ of plant (db)].

Extract preparation by conventional maceration (CM) and ultrasound-assisted extraction (UAE)

Fresh plant leaves were extracted with $70 \%$ ethanol at a ratio between fresh plants: ethanol of 1:5. The mixture was left for 12 days macerating at room temperature $\left(20^{\circ} \mathrm{C}\right)$ in dark conditions. The solids were then separated by vacuum filtration through a ceramic filter with a porosity of 40 $\mu \mathrm{m}$, and the extracts were stored at $4^{\circ} \mathrm{C}$ until their use in the following analysis. To avoid degradation of bioactive compounds, an ultrasonic bath (Transsonic T310, Elma, Singen, Germany) was used as an ultrasound source (frequency $35 \mathrm{kHz}$ ) (Aspé and Fernández, 2011). The ultrasound-assisted extraction was performed under ambient conditions $\left(20-25^{\circ} \mathrm{C}\right)$ for $15 \mathrm{~min}$ in cycles over two hours. The crude extracts were stored at $0-4^{\circ} \mathrm{C}$ until analysis.

\section{Determination of total polyphenol content (TP)}

The total polyphenol content was determined by a spectrophotometric method, using gallic acid as a standard, according to the method described by the International Organization for Standardization ISO14502-1 (Anesini et al., 2008; Miron et al., 2010). In a short description of the method, $0.1 \mathrm{~mL}$ of each extract was transferred into glass tubes, then $5 \mathrm{~mL}$ of Folin-Ciocâlteu's reagent diluted 1/10 in distillated water, and $4 \mathrm{~mL}$ of a sodium carbonate solution $(7.5 \% \mathrm{w} / \mathrm{w})$ were added. All the experiments were run in duplicate, and allowed to stand at room temperature for one hour before the absorbance was determined at 765 $\mathrm{nm}$ against a blank containing distilled water. A standard curve of gallic acid (ranging from 10 to $50 \mu \mathrm{g}$ gallic acid/ $\mathrm{mL}$ and $\mathrm{R}^{2}=0.9988$ ) was used to express the concentration of polyphenols in samples as gallic acid equivalents (GAE) in $\mathrm{g} /(100 \mathrm{~g}$ dry basis of material).

\section{Estimation of total flavonoid content}

The total flavonoid content in the extracts was determined by a spectrophotometric method based on the formation of complex flavonoid-aluminium with an absortivity maximum between 420-430 nm (Bozin et al., 2008; Jia et al., 1999). Briefly, $1 \mathrm{~mL}$ of each extract was separately mixed with $1 \mathrm{~mL}$ of $2.5 \% \mathrm{AlCl}_{3} \cdot 6 \mathrm{H}_{2} \mathrm{O}, 2 \mathrm{~mL}$ of $10 \%$ sodium acetate solution, and $70 \%$ ethanol to a total volume of $10 \mathrm{~mL}$. The experiments were run in duplicate, and after incubation at room temperature for 30 minutes, the absorbance of the reaction mixtures was measured at 420 $\mathrm{nm}$ against a water standard. The flavonoid content values were determined from a standard curve prepared with quercetin (ranging from 10 to $50 \mu \mathrm{g} / \mathrm{mL}$ final volume and $\mathrm{R}^{2}=0.9732$ ) and expressed as mg quercetin equivalents (QE)/kg plant.

\section{Statistical analysis}

The experimental results were analysed using Principal Component Analysis (PCA) with full cross-validation. PCA constitutes the most basic statistical method of all multivariate data analyses, and involves decomposing one "data matrix" into a structural part (model) and a "noise" part (error). The main propose of all multivariate data analysis is to decompose the data in order to detect and model "hidden phenomena". PCA was assessed using the Unscrambler X 10.1 software version from CAMO Software AS (Oslo, Norway). PCA was used to determine the influence of extraction method and type of Allium spp. on the total polyphenol and flavonoid content of the extracts obtained.

\section{Results and discussion}

\section{Physicochemical properties of raw plant material}

This study is based on the fact that, in Romania during to the spring season, an increased consumption of onion and wild garlic leaves as dietary supplements occurs. Thus, it was also took into account the growing interest in the development of agronomical area of the raw materials selected. Therefore, particular attention was given to the initial physical and physicochemical characteristics presented in Tab. 1. For Allium cepa varieties, the $\mathrm{pH}$ values were between 5.7-5.9. Other reports have present for some Span- 
ish onion variety the same values for $\mathrm{pH}$ (Roldán-Marín $e$ t al., 2009). The crude fat content may suggest, in the case of the Allium ursinum and Allium cepa varieties, the possibility of using them to obtain essential oils useful for making dishes more flavor, or for encapsulation/formulation processes.

Bioactive composition of onion and wild garlic leaves (total polyphenols and quercetin content)

The bioactive compositions of three varieties of Allium spp. leaf extracts were analyzed for total phenolic and flavonoid contents using different methods of extraction. It has been reported that onion is one of the major sources of dietary flavonoids present as glycosides or as aglycones (Corzo-Martínez et al., 2007). Sellappan and Akoh (2002) identified the major flavonoid compound in onion as quercetin, present in conjugated form as quercetin 4'-O- $\beta$ glycopyranoside, quercetin 3,4'-O- $\beta$-diglycopyranoside, and quercetin 3,7,4'-O- $\beta$-triglycopyranoside.

The bioactive compounds from different Allium spp. plants have also been confirmed by other literature reports. Some authors previously identified 3, 4' $-O$-diglucoside and $4^{\prime}-O$-monoglucoside as the major quercetin derivatives of the mature red onion bulb; these components account for about $93 \%$ of the total flavonols (Pérez-Gregorio et al., 2011). Rodriguez et al. (2009) affirmed that different treatments (curing and cooking) of onion bulbs could influence their flavonol and anthocyanin levels, and demonstrated that intense microwave cooking treat-

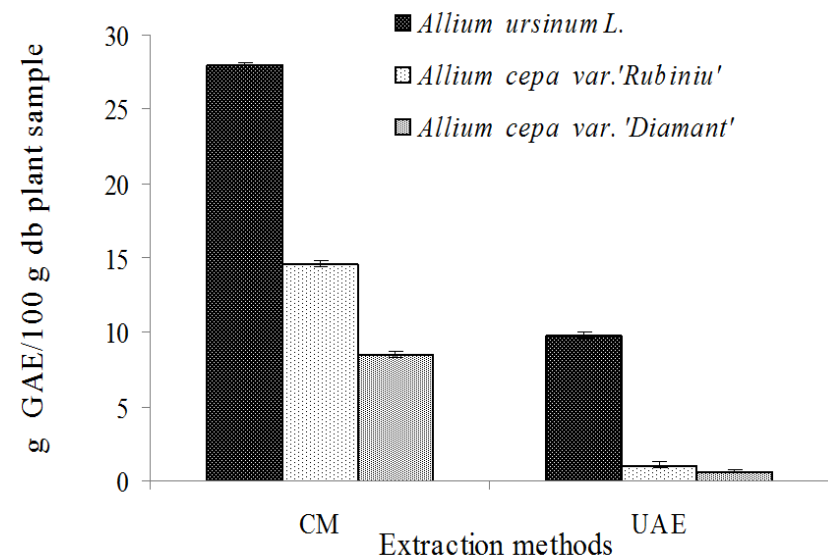

Fig. 1. Polyphenol content of onion and wild garlic leaf extracts. ( ${ }^{*}$ Data expressed as means \pm standard deviations of duplicate analyses of each extraction method; CM-conventional maceration; UAE-ultrasound-assisted extraction; db-dry basis)

Tab. 1. Initial physical and physicochemical characteristics of onion and wild garlic leaves

\begin{tabular}{cccc}
\hline Characteristic* & Allium cepa var. 'Diamant' & Allium cepa var. 'Rubiniu' & Allium ursinum L. \\
\hline dry matter, \% & $5.23204 \pm 0.088672$ & $6.48318 \pm 0.0253$ & $8.95453 \pm 0.26351$ \\
moisture, \% & $94.76795 \pm 0.0724$ & $93.5168 \pm 0.0051$ & $91.04546 \pm 0.47269$ \\
ash content, \% (db) & $9.32582 \pm 0.02075$ & $9.08414 \pm 0.1116$ & $8.77613 \pm 0.0902$ \\
crude fat content, \% & $2.3944 \pm 0.2354$ & $2.48053 \pm 0.0869$ & $3.61355 \pm 0.0778$ \\
pH & $5.93 \pm 0.0565$ & $5.75 \pm 0.0353$ & $6.235 \pm 0.16263$ \\
\hline $\begin{array}{c}\text { titratable acidity } \\
\text { gacid citric/100 g plant (db) }\end{array}$ & $0.8884 \pm 0.10784$ & $1.19856 \pm 0.0521$ & $0.86734 \pm 0.0019$ \\
\hline
\end{tabular}

\footnotetext{
${ }^{*}$ Values are the mean of two independent determinations \pm standard deviations; db: dry basis
}

$3,4^{\prime}$-diglucoside and quercetin $4^{\prime}$-glucoside, respectively.

In order to investigate the impact of different extraction methods on the content of bioactive compounds, selected materials from each plant type were obtained using two different types of extracts: conventional maceration, and ultrasound-assisted extraction. The amount of total polyphenolics varied widely with the extraction method and source of plant materials. The highest total polyphenolic content (Fig. 1), with a conventional maceration extract from Allium ursinum L. leaves, was determined to be $27.8884 \mathrm{~g} \mathrm{GAE} / 100 \mathrm{~g}$ dry basis.

The level of total polyphenols was much higher ( $57.74 \%$ ) with a 'Rubiniu' variety extract obtained by conventional maceration, and $58.64 \%$ higher with ultrasoundassisted extraction, respectively. Gökçe Fuat et al. (2010) reported that the red onion had a higher equivalent gallic acid content than the white onion (2.2 $\mathrm{mg} \mathrm{GAE} / \mathrm{g}$ ). Significant differences in flavonoid concentrations (Fig. 2) were obtained between varieties. Most studies have reported higher levels of flavonoids in red onion than in other onion varieties (Lin and Tang, 2007; Rodrigues et al., 2011) but the analysis were done on bulbs.

The results indicate that ultrasound-assisted extraction is a good method for obtaining the highest flavonoid content, for all the raw materials used. Consequently, the total flavonoid content was lower in the white variety 'Diamant', than in the red variety 'Rubiniu', ranging from $5.4867 \mathrm{mg}$ $\mathrm{QE} / \mathrm{kg}$ fresh plant to $6.0304 \mathrm{mg} \mathrm{QE} / \mathrm{kg}$ fresh plant. Instead, the highest flavonoid content was identified in $\mathrm{Al}$ lium ursinum L. leaves, $7.2730 \mathrm{mg} \mathrm{QE} / \mathrm{kg}$ fresh plant. The present results are in agreement with other reports that indicate a higher quercetin content in red onions than in yellow or white onions (Nemeth and Piskula, 2007). Pérez-Gregorio et al. (2010) determined the flavonol and anthocyanin concentrations in different varieties of red and white onions. They found that flavonols (quercetin 7,4-diglucoside, quercetin 3,4-diglucoside, quercetin 3 -glucoside, quercetin 4-glucoside) were the predominant polyphenolic compounds. White onion cultivars had the lowest total flavonol content $(89.3 \pm 38.5$ and $101.0 \pm 18.9$ $\mathrm{mg}$ quercetin $/ \mathrm{kg}$ fresh weight for 'Braca da Póvoa' and the hybrid 'SK 409 '), whereas red onions presented the highest levels of flavonols, with $280.2 \pm 41.5$ and $304.3 \pm 81.2$ $\mathrm{mg}$ quercetin $/ \mathrm{kg}$ fresh weight for 'Vermelha da Póvoa' and 'Red Creole', respectively.

Values are the mean of two independent determinations \pm standard deviations, db. dry basis 


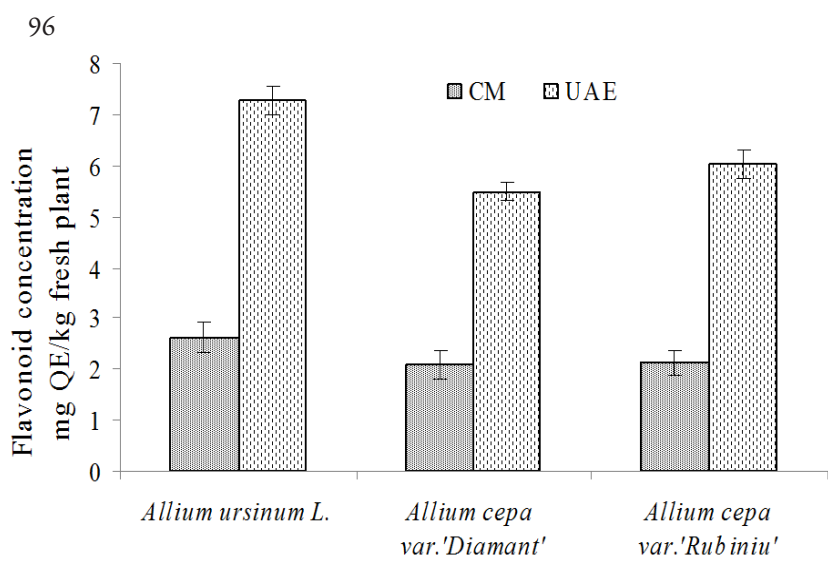

Fig. 2. Flavonoid contents of wild garlic, white, and red onion leaf extracts. ( ${ }^{*}$ Error bars represent standard deviations for two duplicate determinations)

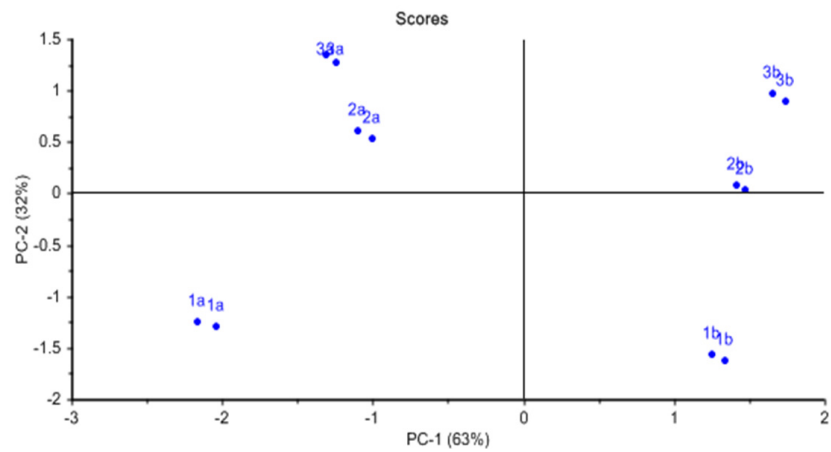

Fig. 3. Score plot after PCA of the individuals in the plane defined by the two first PCs. 1-Allium ursinum L; 2-Allium cepa var. 'Diamant', 3-Allium cepa var. 'Rubiniu'; a-conventional maceration; b-ultrasound-assisted extraction

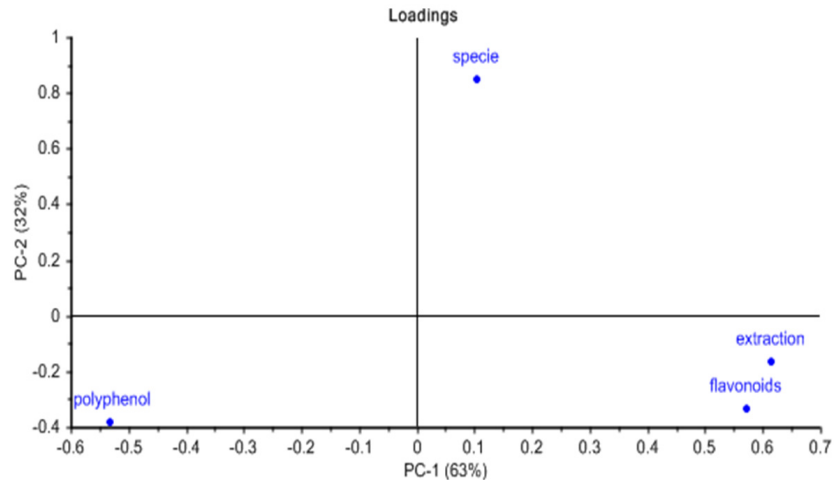

Fig. 4. Loading plot after PCA with full cross-validation of the analysed samples

A principal component analysis (PCA) with full cross-validation was carried out in order to evaluate the influence of plant species and extraction method on the concentrations of bioactive compounds. PCA is a linear transformation of a set of original data to a set of uncorrelated components in such a way that only a few of the resulting variables account for the majority of the variability observed in the original data. Therefore, a reduction in dimensionality is achieved with minimal loss of information (Calvo et al., 2010). Visualization of the results of PCA is usually achieved by plotting pairs of the first few principal components (PCs). In this study, Principal Component
1 (PC1) and Principal Component 2 (PC2) represented $94.93 \%$ of the system variance. Principal component 2 (PC2) is the species analysed, but the most important influence (63\%), as indicated by Principal Component 1 (PC1), represented the extraction method used in the experiment; that was of significant importance for the variations in concentrations of bioactive compounds, total polyphenols, and flavonoids. Fig. 3 and 4 represent the score plots (maps of analysed samples) and loading plots (plot of variables) for all the Allium spp. and extraction methods used in the experiment.

It can also be observed that the extraction method significantly influenced the type of bioactive compounds extracted. Using the conventional maceration extraction method (range 1), higher amounts of polyphenols were extracted, while after the ultrasound-assisted extraction method (range 2), concentrations of flavonoids were much higher. The type of Romanian Allium spp. did not influence the overall amount of bioactive components extracted.

\section{Conclusions}

The obtain results showed that Allium ursinum leaves contained the highest amounts of oil (3.61\%) and dry matter (8.95\%), and pH (6.235). Comparatively with Allium cepa var 'Diamant', all physicochemical properties are much higher in the Allium cepa var. 'Rubiniu', with $27.59 \%$ dry matter and $31.30 \%$ oil content. The high crude fat content suggests that wild garlic is a good source of aromatic oils in steam distillation processes. In conclusion, and considering the harvest period of the vegetal products, the polyphenol content of the analyzed extracts is influenced by the type of processing method the samples undergo. The results obtained were statistically validated and compared with data in the scientific literature. This study may be considered a new report about the identification and characterization of bioactive compounds from Romanian fresh Allium spp. leaves as natural and important sources that are consumed, especially, during the spring season.

\section{Acknowledgements}

This work benefited from financial support through the 2010 POSDRU/89/1.5/S/52432 project, Organizing the National Interest Postdoctoral School of Applied Biotechnologies with Impact on Romanian Bioeconomy, a project co-financed by the European Social Fund through the Sectoral Operational Programme Human Resources Development 2007-2013.

\section{References}

Anesini C, Ferraro GE, Filip R (2008). Total polyphenol content and antioxidant capacity of commercially available tea (Camellia sinensis) in Argentina. J Agr Food Chem 56:9225-9229.

AOAC (2000). Official Methods of Analysis no $985.26\left(17^{\text {th }}\right.$ 
Ed). Washington. Assoc of Official Analytical Chemists.

Aspé E, Fernández K (2011). The effect of different extraction techniques on extraction yield, total phenolic and antiradical capacity of extracts from Pinus radiata Bark. Ind Crop Prod 34:838-844.

Bozin B, Mimica-Dukic N, Samojlik I, Goran A, Igic R (2008). Phenolics as antioxidants in garlic (Allium sativum L., Alliaceae). Food Chem 111:925-929.

Calvo P, Hernández T, Lozano M, González-Gómez D (2010). Microencapsulation of extra-virgin olive oil by spray-drying: Influence of wall material and olive quality. Eur J Lipid Sci Technol 112:852-858

Corzo-Martínez M, Corzo N, Villamiel M (2007). Biological properties of onion and garlic. Trends Food Sci Tech 18:609625.

Dini I, Tenore GC, Dini A (2008). Chemical composition, nutritional value and antioxidant properties of Allium caepa L. Var. 'Tropeana' (red onion) seeds. Food Chem 107:613621.

Djurdjevic L, Dinic A, Pavlovic P, Mitrovic M, Karadzic B, Tesevic V (2004). Allelopathic potential of Allium ursinum L. Biochem Syst Ecol 32:533-544.

FRD Center Market Report (2011). FRD Center Market Entry Services, Demo Report of the Romanian Agribusiness Market. Available at http://www.market-entry.ro.

Gennaro L, Leonardi C, Esposito F, Salucci M, Maiani G, Quaglia G (2002). Flavonoid and carbohydrate contents in Tropea red onions: Effects of homelike peeling and storage. J Agr Food Chem 50:1904-1910.

Gökçe Fuat A, Cemal K, Serçe S, Özgen M (2010). Effect of scale color on the antioxidant capacity of onions. Sci HorticAmsterdam 123:431-435.

Gorinstein S, Jastrzebski Z, Namiesnik J, Leontowicz H, Leontowicz M, Trakhtenberg S (2007). The atherosclerotic heart disease and protecting properties of garlic: Contemporary data. Mol Nutr Food Res 51:1365-1381.

Hiyasat B, Sabha D, Grotzinger K, Kempfert J, Rauwald JW, Mohr FW, Dhein S (2009). Antiplatelet activity of Allium ursinum and Allium sativum. Pharmacol 83:197-204.

Hodge G, Hodge S, Han P (2002). Allium sativum (garlic) suppresses leukocyte inflammatory cytokine production in vitro: Potential therapeutic use in the treatment of inflammatory bowel disease. Cytometry 48:209-215.

Jia Z, Tang M, Wu J (1999). The determination of flavonoid content in mulberry and scavenging effect on superoxide radicals. Food Chem 64:555-599.

Kalayarasan S, Prabhu PN, Manikandan R, Arumugam M, Sudhandiran G (2009). Diallyl sulfide enhances antioxidants and inhibits inflammation through the activation of Nrf2 against gentamicin-induced nephrotoxicity in Wistar rats. Eur J Pharacol 606:162-171.

Lanzotti V (2006). The analysis of onion and garlic. J Chromatogr A 1112:3-22.

Lin CY, Tang CY (2007). Determination of total phenolics and flavonoid contents in selected fruits and vegetables, as well as their stimulatory effects on mouse splenocyte proliferation. Food Chem 101:140-147.

Miron LT, Gazi I, Plaza del Moral M (2010). Romanian aromatic plants: as sources of antioxidants. Innovative Romanian Food Biotechnol 6:18-24.

Najjaa H, Neffati M, Zouari S, Ammar E (2007). Essential oil composition and antibacterial activity of different extracts of Allium roseum L., a North African endemic species. C R Chimie 10:820-826.

Nemeth K, Piskula MK (2007). Food content, processing, adsorbtion and metabolism of onion flavonoids. Crit Rev Food Sci 47:397-409.

Pérez-Gregorio MR, Regueìro J, González-Barreíro C, RíalOtero R, Símal-Gándara J (2011). Changes in antioxidant flavonoids during freeze-drying of red onions and subsequent storage. Food Control 22:1108-1113.

Pérez-Gregorio RM, García-Falcón MS, Símal-Gándara J Rodrigues AS, Almeida Domingos PF (2010). Identification and quantification of flavonoids in traditional cultivars of red and white onions at harvest. J Food Compos Anal 23(6):592-598.

Rodrìgues AS, Pérez-Gregorio MR, García-Falcón MS, SimalGándara J, Almeida DPF (2011). Effect of meteorological conditions on antioxidant flavonoids in Portuguese cultivars of white and red onions. Food Chem 124:303-308.

Rodrìgues AS, Pérez-Gregorio RM, García-Falcón MS, SímalGándara J (2009). Effect of curing and cooking on flavonols and anthocyanins in traditional varieties of onion bulbs. Food Res Int 42(9):1331-1336.

Roldán-Marín E, Sánchez-Moreno C, Rosana L, Begoňa de Ancos, Cano MP (2009). Onion high-pressure processing: Flavonol content and antioxidant activity. LWT-Food Sci Technol 42:835-841.

Santas J, Carbó R, Gordon MH, Almajano MP (2008). Comparison of the antioxidant activity of two Spanish onion varieties. Food Chem 107:1210-1216.

Sellappan S, Akoh CC (2002). Flavonoids and antioxidant capacity of Georgia-grown Vidalia onions. J Agr Food Chem 50:5338-5342.

Sengupta A, Ghosh S, Bhattacharjee S (2004). Allium vegetables in cancer prevention: An overview. Asian Pac J Cancer Prev $5: 237-245$.

Shukla Y, Kalra N (2007). Cancer chemoprevention with garlic and its constituents. Cancer Lett 247:167-181.

Siegel G, Malmsten M, Pietzsch J, Schmidt A, Buddecke E, Michel F (2004). The effect of garlic on arteriosclerotic nanoplaque formation and size. Phytomedicine 11:24-35.

Takahashi M, Shibamoto T (2008). Chemical compositions and antioxidant/anti-inflammatory activities of steam distillate from freeze-dried onion (Allium cepa L.) sprout. J Agr Food Chem 56:10462-10467.

Tepe B, Sokmen M, Akpulat Askin H, Atalay S (2005). In vitro antioxidant activities of the methanol extracts of five Allium species from Turkey. Food Chem 92:89-92. 